\title{
Star and Stellar Cluster Formation: ALMA-SKA Synergies
}

\section{G. A. Fuller}

Jodrell Bank Centre for Astrophysics \& UK ALMA Regional Centre Node, School of Physics \& Astronomy, University of Manchester, UK

E-mail: G.Fuller@manchester.ac.uk

\section{J. Forbrich}

Institute of Astronomy, University of Vienna, Austria

E-mail: jan.forbrich@univie.ac.at

\section{J. M. Rathborne}

CSIRO Astronomy and Space Science, Australia

E-mail: Jill.Rathborne@csiro.au

\section{S. Longmore}

Astrophysics Research Institute, Liverpool John Moores University, UK

E-mail: S.N.Longmore@ 1 jmu.ac.uk

\section{S. Molinari*}

INAF-IFSI, Rome, Italy

E-mail: molinarieiaps.inaf.it

Over the next decade, observations conducted with ALMA and the SKA will reveal the process of mass assembly and accretion onto young stars and will be revolutionary for studies of star formation. Here we summarise the capabilities of ALMA and discuss recent results from its early science observations. We then review infrared and radio variability observations of both young low-mass and high-mass stars. A time domain SKA radio continuum survey of star forming regions is then outlined. This survey will produce radio light-curves for hundreds of young sources, providing for the first time a systematic survey of radio variability across the full range of stellar masses. These light-curves will probe the magnetospheric interactions of young binary systems, the origins of outflows, trace episodic accretion on the central sources and potentially constrain the rotation rates of embedded sources.

\section{Advancing Astrophysics with the Square Kilometre Array}

June 8-13, 2014

Giardini Naxos, Italy

\footnotetext{
*Speaker.
} 


\section{Introduction}

The Atacama Large Millimeter/submillimeter Array (ALMA) ${ }^{1}$ is the largest telescope yet constructed. Initial results from its early science observations span the range from the detection of a remarkable spiral patterned mass loss resulting from the interaction of an ABG star and its binary companion (Maercker et al. 2012) to constraining the density of extreme starburst galaxies at $z>1$ (Karim et al. 2013). Here we focus on galactic star formation and the synergies provided by the complementarity of ALMA and SKA observations.

For studies of star formation, ALMA observations will trace how cold dust and gas is assembled on the small-scales within a molecular cloud and how disks around young stars are formed. SKA will be essential for complementing these studies by tracing the higher energy phenomena associated with the accretion of this material on to the star, probing the stellar and disk magnetospheres, and the ionising feedback from outflows and stellar winds. Combined, these cutting edge facilities will reveal the process of mass assembly and accretion onto young stars and will be revolutionary for studies of star formation.

\section{Capabilities: ALMA and SKA}

Comprising fifty four $12 \mathrm{~m}$ diameter dishes plus twelve $7 \mathrm{~m}$ diameter dishes, ALMA is an international collaboration between ESO, NRAO and NAOJ presenting Europe, North America and East Asia respectively in cooperation with the Republic of Chile. Operating between $30 \mathrm{GHz}$ and $900 \mathrm{GHz}(10 \mathrm{~mm}-0.35 \mathrm{~mm})$ in 10 frequency bands which span the available atmospheric windows to study the emission from cold dust and molecular gas, ALMA is located on the $5000 \mathrm{~m}$ altitude Chajnantor plateau in northern Chile.

There were two key science drivers for the design of ALMA. The first is the imaging of the physical, chemical and magnetic structure of protostellar/protoplanetary disks around low mass protostars in the nearest star forming regions. The second is imaging of Milky Way-mass galaxies at $z=3$ in $\mathrm{CO}$ or $\mathrm{C}^{+}$(the brightest tracers of molecular gas) in less than 24 hours. In parallel with these is the requirement to routinely deliver high precision images at an angular resolution of 0.1 ". With $8 \mathrm{GHz}$ of instantaneous bandwidth, the ALMA correlator provides spectral resolutions up to maximum of $3.8 \mathrm{kHz}$, corresponding to a velocity resolution of $0.01 \mathrm{~km} / \mathrm{s}$ at $110 \mathrm{GHz}$. In full operation ALMA with reach continuum noise levels of $1 \mathrm{mJy}$ (at $110 \mathrm{GHz}$ ) in 1 second, corresponding to a mass of $\sim 0.004 \mathrm{M}_{\odot}$ per beam in the nearest star forming regions and $\sim 5 \mathrm{M}_{\odot}$ at $5 \mathrm{kpc}$. Figure 1 shows the angular size scale versus frequency coverage of ALMA compared with SKA1-Mid (and the NRAO Karl G. Jansky Very Large Array, JVLA). As the figure demonstrates, to probe similar angular scales as ALMA at frequencies above $60 \mathrm{GHz}$, requires frequency Band 5 on SKA1-Mid. As an indication of the range of physical scales relevant for studies of galactic star formation, the horizontal lines on the figure show the angular size required to image a hypercompact HII region, the earliest phase of massive star formation where ionised gas is detected, and a large circumstellar disk/toroid at the typical distance to molecular clouds in the molecular ring, $\sim 5 \mathrm{kpc}$.

\footnotetext{
${ }^{1}$ www.almaobservatory.org
} 


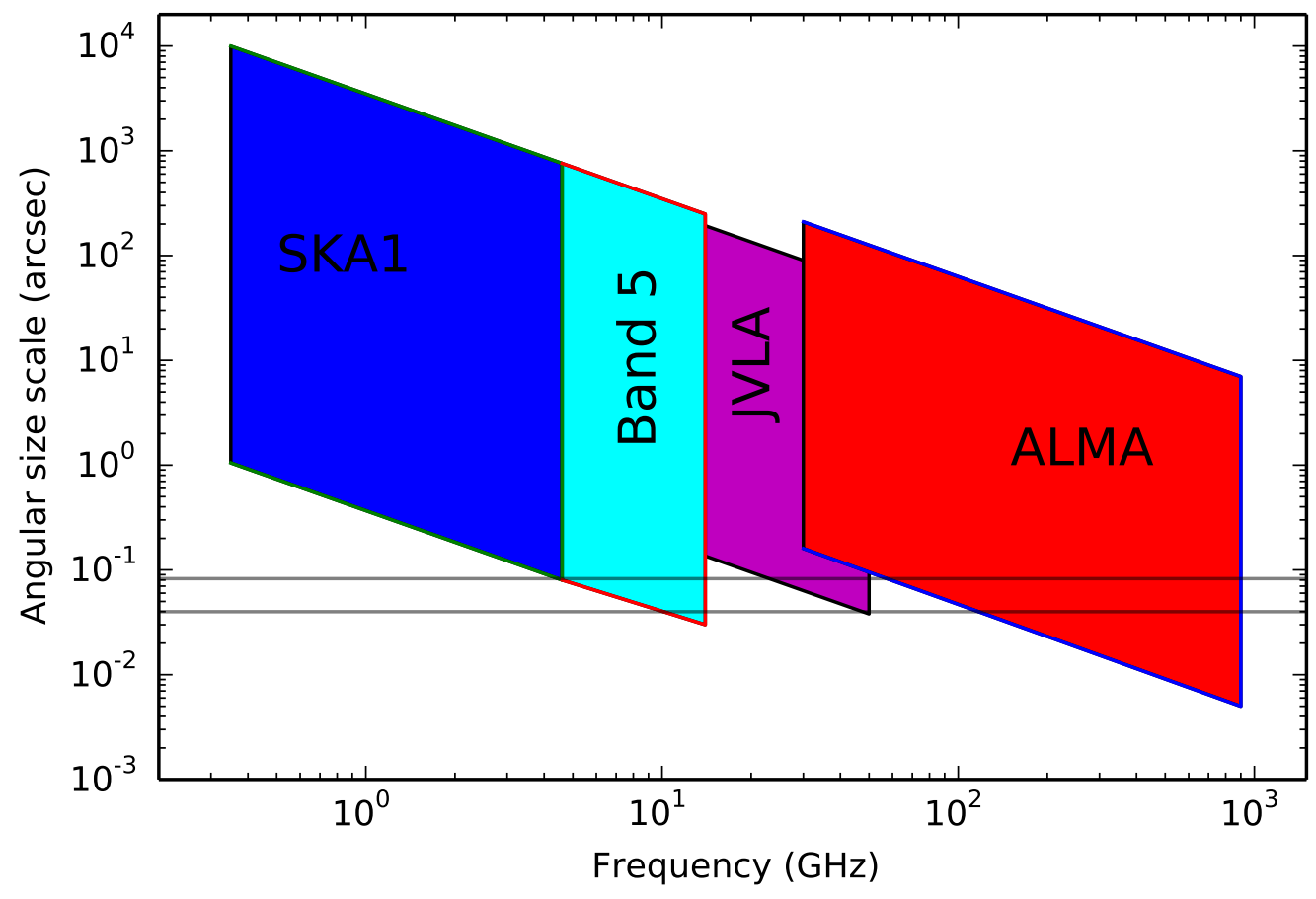

Figure 1: Angular size scale as a function of frequency for SKA1, JVLA and ALMA. Frequency band 5 on SKA1-Mid is identified separately. The shaded regions show the angular scale from the maximum resolution at each frequency to the instantaneous field of view. Larger angular size scales are of course accessible through mosaicing. The two horizontal lines indicate the resolution required to resolve a $0.01 \mathrm{pc}$-sized hypercompact HII region (upper) and a $1000 \mathrm{AU}$ circumstellar disk/toroid (lower) at a distance of $5 \mathrm{kpc}$.

\section{Tracing global collapse, disks and outflows}

The ultimate fate of infalling material, and how stars actually gain mass, depends on the processes taking place on small spatial scales close to a forming star. Recent evidence suggests that the final stages of accretion on to the central star may be highly episodic. For example, ALMA observations of the chemical composition of the envelope around a low mass protostar shows evidence of heating associated with a burst of accretion 100-1000 years ago when the mass accretion rate was a factor of $\sim 100$ above its current rate (Jørgensen et al. 2013).

On the large spatial scales, ALMA observations of the massive infrared dark cloud SDC335 (Peretto et al. 2013) show that the infalling dense gas seen in single dish images is confined to a number of filaments, allowing us to trace the large-scale global collapse. On the small scales, ALMA observations of high excitation lines of both complex organic species (Pineda et al. 2012) and simple species (Zapata et al. 2013) show infall profiles towards one component of the low mass protostellar binary IRAS16293. Indeed, measuring infall is a crucially important tool for understanding star formation: line profiles diagnostic of infalling gas (e.g. Anglada et al. 1987) have been identified towards starless cores (e.g. Lee et al. 1999), low mass protostars (e.g. Walsh et al. 2006), high mass young stellar objects (e.g. Fuller et al. 2005), UCHII regions (e.g. Ho \& 
Young 1996), and molecular filaments (e.g. Kirk et al. 2013).

Circumstellar disks are the mass reservoir out of which planets form. ALMA observations are elucidating the structure and composition of these disks with unprecedented detail. Observations of transition disks, relatively evolved disks with larger inner holes, have revealed large azimuthal asymmetries in the dust continuum emission at radii of $\sim 30-50 \mathrm{AU}$ (Casassus et al. 2013; van der Marel et al. 2013; Pérez et al. 2014). These asymmetries are interpreted as resulting from the trapping of dust in large anticyclonic vortices in the disk.

The growth of dust grains is a critical step towards planet formation which SKA will probe. Regions of disks where this growth may be occuring are revealed by ALMA observations, not only of the dust continuum emission, but also using molecular lines to trace the $\mathrm{CO}$ snowline, where $\mathrm{CO}$ condensed on to grain surfaces (Qi et al. 2013; Mathews et al. 2013). ALMA observations also reveal evidence for complex chemistry in a transition disk (van der Marel et al. 2014) as well the presence of a simple sugars (Jørgensen et al. 2012). Future SKA observations will be critical for the identification of complex organic species towards these objects.

Material in protoplanetary disks can be eroded by the winds and high energy radiation from nearby high-mass stars. To quantify this interaction, ALMA observations can probe molecular gas in the disk (Mann et al. 2014) while SKA can probe the photoionized gas flow from the disk as well as the interaction zone between the gas and the impinging stellar wind (e.g. Graham et al. 2002).

Outflows are an ubiquitous feature of star formation. Driven by winds and jets which originate from the inner disk/disk-star interface region (Li et al. 2014), they not only sculpt, and eventually clear the material around the protostar, but also inject energy into the surrounding medium and remove angular momentum. The bulk of the molecular line emission seen from outflows detected via ALMA observations (e.g. Arce et al. 2013) will trace material swept up by the stellar wind. The nature of the jets from stars evolves as the driving source evolves (Frank et al. 2014) but observations show the jets have a significant atomic component potentially observable by SKA in HI.

\section{Accretion and Variability in Young Stars}

Low-mass young stellar objects (YSOs) are thought to accrete mass via their circumstellar disks. Associated accretion shocks can be observed both when mass is accreted onto the disk and also when it is eventually accreted onto the central object (e.g. Hartmann 2009). The accretion process is observable at wavelengths ranging from the radio to the X-ray regime, including optical and infrared wavelengths. The connection of accretion and high-energy processes (e.g. Feigelson \& Montmerle 1999) detected in both X-ray and radio emission has been highlighted by spectacular examples like V1647 Ori (Kastner et al. 2004), while spectral lines in the X-ray can provide an estimate of the the accretion rate (Brickhouse et al. 2012).

In the centimeter regime, accretion processes can be associated with both thermal (free-free) and nonthermal (gyrosynchrotron) radiation, as discriminated by their spectral and polarization properties. Generally, it is thought that the nonthermal radiation emanates from the magnetospheric structures in the innermost vicinity of YSOs while thermal emission most likely traces the bases of outflows and jets further away from the central object. 
Recently there has been considerable observational progress in two relevant areas. First at centimeter wavelengths, new instrumentation, provided via the expanded capabilities of the JVLA, is rekindling protostellar radio astronomy. In tandem with these advances, a multi-wavelength, timedomain view of the dynamic processes in YSOs, including accretion is emerging. Well sampled time series datasets are now becoming available, in particular in the infrared. Simultaneously obtained multi-wavelength time series datasets are also becoming increasingly important to constrain the underlying physical processes.

\subsection{Identifying radio counterparts to low-mass YSOs}

Observations with the VLA initiated protostellar radio astronomy by providing excellent angular resolution to reliably identify radio counterparts to low-mass YSOs. While the first radiodetected YSOs identified by the VLA were found in the Orion Nebula Cluster (e.g. Moran et al. 1983; Garay et al. 1987), the radio sample remained incomplete, as indicated by the fraction of radio detected YSOs being consistently lower than the X-ray detection fraction (Forbrich \& Wolk 2013).

Prior to the sensitivity upgrade of the VLA, only the most nearby star-forming regions could be studied in detail. The low-mass Coronet cluster at a distance of $130 \mathrm{pc}$ is an example where almost all known YSOs in the inner cluster have radio counterparts (Brown 1987; Forbrich et al. 2006). This region was shown to host the first of only a few known protostars with confirmed nonthermal radio emission as inferred from circular polarization indicative of gyrosynchrotron emission (Feigelson et al. 1998; Choi et al. 2009).

The newly upgraded JVLA has already significantly advanced this research by providing a more complete census of YSOs detected at centimetre wavelengths (Dzib et al. 2013b; Kounkel et al. 2014). Indeed, with the improved JVLA, a more complete census of YSOs in nearby starforming regions is emerging, both in terms of sensitivity and in area covered. Using rapid variability, negative spectral indices and polarization as indicators, a recent, large survey of $\rho$ Oph revealed that about half of the identified YSOs show nonthermal emission (Dzib et al. 2013b).

Similarly, a recent large-scale (more than 2 square degrees) survey of the Orion Nebula Cluster, detected 374 sources at $4.5 \mathrm{GHz}$ (Kounkel et al. 2014). Of these, 148 had been previously classified as YSOs and 86 additional sources are inferred to be new YSO candidates. With reliably determined spectral indices, these sources will be used as targets for follow-up VLBI observations to study parallaxes and proper motions. New and sensitive monitoring observations of the Coronet cluster show that the youngest sources in the sample are the brightest and least variable, possibly exhibiting mostly thermal wind emission (Liu et al. 2014).

\subsection{Time variability}

The significant increase in sensitivity provided by new JVLA observations is also starting to produce radio light-curves for YSOs. While YSO X-ray flares have been known for some time (e.g. Getman et al. 2008), very little is known about protostellar radio flares (Bower et al. 2003; Forbrich et al. 2008) and a possible X-ray-radio connection (e.g. Guedel \& Benz 1993; Forbrich \& Wolk 2013). As a result the physics of YSO radio flares is not currently well understood although they are thought to be produced by coronal-type activity in scenarios that also produce X-ray emission 
(e.g. Drake et al. 1992). Some of the activity could also be directly or indirectly related to accretion. However, some YSO flares are thought to be due to the interaction of large magnetospheric features in close binary systems (e.g., V773 Tau: Massi et al. (2006) and DQ Tau: Salter et al. (2008); Getman et al. (2011) and references therein) or from periodic accretion bursts (Muzerolle et al. 2013; Balog et al. 2014; Bary \& Petersen 2014).

Impressive time domain studies are now being carried out in the infrared. A 30-day Spitzer and CoRot photometric monitoring campaign of more than a thousand YSOs in NGC 2264 (Cody et al. 2014) classified a variety of different variability mechanisms in YSOs, mainly on timescales of days. Other recent work suggests that short-term optical variability may be due to enhanced accretion activity (Stauffer et al. 2014), while mid-infrared variability may be due to structural perturbations in the inner disk (Flaherty et al. 2013). Spectroscopic monitoring campaigns have also revealed a new level of complexity. Multi-epoch near-infrared spectroscopy of several accreting YSOs in $\rho$ Oph observed as part of the Spitzer YSOVAR project, showed no correlation between the YSO mid-IR light curves and time-resolved veiling or mass accretion rates (Faesi et al. 2012).

\subsection{Accretion onto high-mass stars}

Strong magnetic fields and circumstellar disks are key drivers of the evolution of, and activity in, the inner circumstellar regions of low mass stars and there is growing evidence these important components are also present in young high-mass, $M>8 M_{\odot}$, stars. Keplerian disks have been detected through high angular resolution imaging of molecular lines around a number of B-type stars (e.g. Cesaroni et al. 2014; Beltrán et al. 2014) where, like their lower mass counterparts, they presumably play a role in the accretion of material on to the central star. Stellar magnetic fields in the range of hundreds to thousands of kG have also been measured towards OB stars (Nazé 2014). Since stellar magnetic fields are expected to decay away as the high-mass star evolves due to the absence of a convective zone, similar or stronger fields are likely present towards younger stars where they can play an important role in the rotational braking of the stars, setting their initial rotation rates (Meynet et al. 2011; Rosen et al. 2012). The presence of disks and strong magnetic fields suggest that young high-mass stars can show a similar range of circumstellar energetic phenomena as seen towards young low-mass stars, probing the final stages of mass inflow on to the central star as well as the launching of outflows.

Indeed non-thermal radio emission has been detected towards an increasing number of young high-mass stars (e.g. Andre et al. 1988; Rodríguez et al. 2012; Moscadelli et al. 2013; Rodríguez et al. 2014). This emission can arise from a range of phenomena including magnetospheric activity (such as due to variations in magnetically mediated accretion on to the central star), interacting winds in a massive binary system and synchrotron emission from a jet, all processes which are likely to be variable on a range of timescales as seen towards low mass sources (e.g. Dzib et al. 2013b). For example, a source in Orion has been seen to increase in flux by factor of 3 increase over 1 hour (Gómez et al. 2008).

Thermal radio emission from young high-mass stars arises from their ionised winds and their self-photoionised disks, envelopes and surroundings in the form of HII regions. The accretion flow into a HII region and on to forming massive stars will change the ionization balance and hence, flux and size of the ionised region. Such inflowing material will be clumpy and so the mass 
accretion variable. This is borne out by simulations which show that the accretion rates onto stars has considerable time variability, with peak rates between 10 and 100 times higher than the timeaveraged values (e.g. Klassen et al. 2012b). This variable inflow results in a flickering of the radio continuum emission of the HII regions around massive stars. The simulations by Galván-Madrid et al. (2011) found that about $10 \%$ of HII regions showed flux variations of $10 \%$ or more in 10 year timescales. A similar result was found by Klassen et al. (2012a). The radio variability of HII regions can therfore probe the structure and variability of the accretion into massive stars.

To date there have only been a very limited number of studies of the radio variability of HII regions. Galván-Madrid et al. (2008) identified a $\sim 45 \%$ decrease in flux (at $5 \mathrm{GHz}$ ) of a hypercompact HII (HCHII) region over a 5 year period due to enhanced accretion, while $10 \%$ of the ultracompact HII (UCHII) regions with Sgr B2 showed significant changes in flux over a timescale of 23 years (De Pree et al. 2014). The variable central source in $\mathrm{W} 3(\mathrm{OH})$ has changed flux by a factor of 5 on timescales of 9 years (Dzib et al. 2013a) which is interpreted as due to changes in the ionised atmosphere of a circumstellar disk possibly due to changes in the accretion through the circumstellar disk.

The masers observed towards many embedded massive stars provide an additional sensitive probe of changes in the contiunuum emission of their exciting sources. For example, towards some sources the $6.7 \mathrm{GHz}$ methanol maser emission varies periodically or quasi-periodically (Goedhart et al. 2014; Szymczak et al. 2014) which may reflect variations in either the free-free background radiation due to changes in the ionization of the circumstellar material or changes in the infrared pump radiation. Both of these effects may arise from periodic accretion from a circumstellar disk.

\section{SKA1 Young Star Variability Survey}

Both low- and high-mass young stars show centimetre radio continuum emission which is variable on a range of timescales from sub-hour to years and decades. This emission traces a range of phemonena associated with accretion, magnetospheric activity, and outflow which link the hot inner circumstellar regions with the infall and outflow of cooler material traced on larger scales by ALMA. SKA time domain continuum surveys of young stars will provide powerful probes of the inner circumstellar regions of young stars, opening new windows on the star formation process. For example, providing the first comprehensive studies of the properties of the episodic accretion in the inner circumstellar regions of forming massive stars.

In order to confirm the association of radio emission with a particular YSO in crowded cluster environments, as well as isolate the emission close to the central star, such a SKA survey will require sub-arcsecond angular resolution. In addition it will require near simultaneous wide multi-frequency coverage (Figure 2) to constrain the spectral index of the emission to discriminate between thermal and non-thermal emission as well as optically thick and optically thin emission. Measurements of the thermal emission in both the optically thick and thin regimes are important to distinguish between changes in size of the HII region and its ionization. Full Stokes synthesis will be important not only for confirmation of the presence of non-thermal emission, but also to allow the separation of thermal and non-thermal components of the emission.

Figure 2 shows the spectral flux distribution of young HII regions as well as thermal wind and synchrotron emission from lower mass stars. To distinguish between the possible emission 


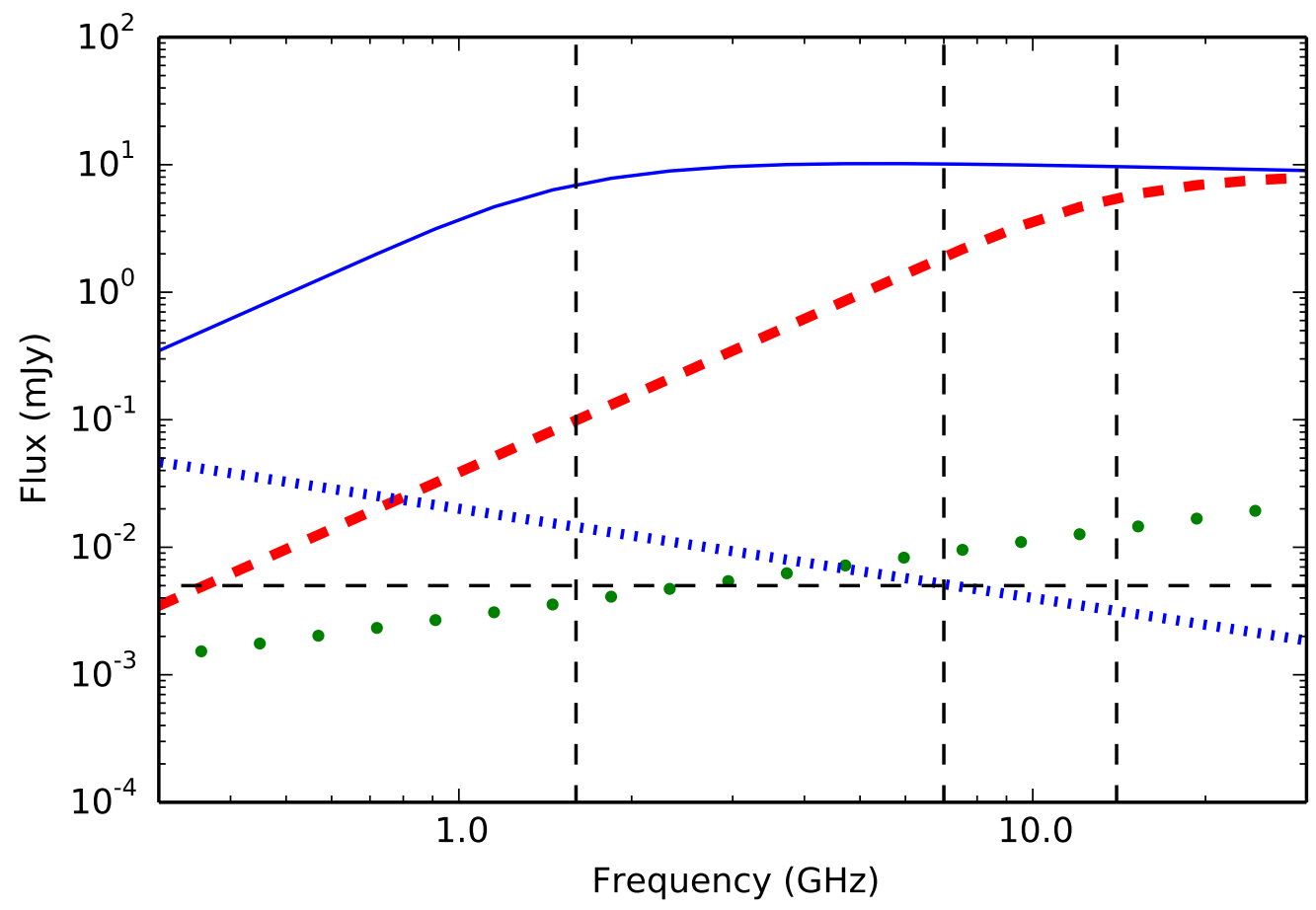

Figure 2: Spectral flux distribution for typical UCHII region (solid, blue curve) with an emission measure of $10^{7} \mathrm{pc} \mathrm{cm}^{-6}$, a typical HCHII region (dashed, red curve) with an emission measure of $10^{9} \mathrm{pc} \mathrm{cm}^{-6}$, a typical ionised wind (with the flux scaling as $v^{0.6}$ ) (green dots) and synchrotron emission $\propto v^{-0.7}$ (dotted, blue curve). The break in the spectrum of the UCHII and HCHII regions marks the transition between optically thin and optically thick emission. The wind and synchrotron emission are normalised to the 4.5 $\mathrm{GHz}$ measured flux of young stellar objects detected in Orion (Kounkel et al. 2014) scaled to a distance of $5 \mathrm{kpc}$. The vertical dashed lines indicate the survey frequencies while the horizontal dashed lines shows the survey $5-\sigma$ noise level $(5 \mu \mathrm{Jy})$.

mechanisms, observations at three frequencies are required. A proposed set of survey frequencies, $1.6 \mathrm{GHz}, 7 \mathrm{GHz}$ and $14 \mathrm{GHz}$, is shown on the figure. As well as providing good sampling of the continuum spectrum of the sources, these particular frequencies also cover several of the key maser transitions (ground state $\mathrm{OH}$ masers at $1.6 \mathrm{GHz}$, Class II methanol masers at $6.7 \mathrm{GHz}$ and $12.2 \mathrm{GHz}$, and the $\mathrm{H}_{2} \mathrm{CO} 2(1,1)-2(1,2)$ transition at $14.5 \mathrm{GHz}^{2}$ ) which are themselves variable, in some cases periodically, and provide an additional, sensitive tracer of changes in the emission from the central sources. A summary of the parameters and the required observing time for a sample survey are given in Table 1.

To sample the range of physical phenomena which give rise to variable radio emission requires repeated observations with a wide range of cadences. Analysis of subsets of the observations will sample sub-hour variability due to flares arising from accretion or magnetospheric events (e.g. Salter et al. 2008; Massi et al. 2006) and rapidly rotating stars (Wolff et al. 2006) while daily to

\footnotetext{
${ }^{2}$ This line is not currently expected to be covered by SKA1 although this would be desirable. However, this frequency is expected to be available with the full SKA.
} 


\begin{tabular}{|c|c|c|c|c|c|}
\hline $\begin{array}{l}\text { Frequency } \\
(\mathrm{GHz})\end{array}$ & $\begin{array}{l}\mathrm{F}_{\mathrm{src}} \\
(\mu \mathrm{Jy})\end{array}$ & $\begin{array}{c}\sigma \\
(\mu \mathrm{Jy})\end{array}$ & $\begin{array}{c}\theta \\
(”)\end{array}$ & $\begin{array}{c}\text { Time } \\
\text { (hours/field) }\end{array}$ & Comments \\
\hline 1.6 & 14 & 1 & 0.5 & 5 & $\begin{array}{l}\text { Optically thick UCHII regions; synchrotron; } \\
\text { OH ground state masers }\end{array}$ \\
\hline 7 & 9 & 1 & 0.5 & 2.5 & $\begin{array}{l}\text { Optically thin UCHII, thick HCHII; } \\
\text { synchrotron-thermal wind cross over; } \\
6.7 \mathrm{GHz} \text { methanol masers }\end{array}$ \\
\hline 14 & 14 & 1 & 0.5 & 2.5 & $\begin{array}{l}\text { Low optical depth } \mathrm{HCHII} \text {; thermal wind } \\
\text { emission; } 12.2 \mathrm{GHz} \text { methanol masers; } \mathrm{H}_{2} \mathrm{CO} \\
\text { transition }\end{array}$ \\
\hline
\end{tabular}

Table 1: SKA1 young star variability survey parameters. The columns show the frequency of observation, the target source flux $\left(\mathrm{F}_{\text {src }}\right)$ and rms flux noise levels $(\sigma)$, the required angular resolution $(\theta)$ and the integration time per field. The comments indidate the emission probed and spectral lines which would be covered in the observations.

monthly observations will sample rotating stars and binary systems with longer periods. Longer observation intervals will sample processes ranging from outflow bursts to changes in the ionization, and therefore flickering, of HII regions due to accretion flows (Galván-Madrid et al. 2011).

The angular resolution of the survey is set by the requirement to isolate individual sources in crowded star forming regions and resolve the emission from individual HCHII regions with sizes $\sim 0.02 \mathrm{pc}$ out to at least the molecular ring at $\sim 5 \mathrm{kpc}$. To connect the detailed studies which can be carried out in nearby star forming regions with the processes in the wider galactic plane requires that similar sources are detectable in both kinds of regions. Therefore the sensitivity required for each single observation is set by the need to sample a similar range of sources to those observed in nearby star forming regions such as Orion at the typical distance of a star forming region in the molecular ring, $5 \mathrm{kpc}$. To detect wind emission sources and synchrotron sources in the Orion star forming region (Kounkel et al. 2014) at $5 \mathrm{kpc}$ implies a required noise level of $1 \mu \mathrm{Jy}$ at each of the observing frequencies (Fig. 2) which should provide detections of up to few hundred sources per star forming region.

Combining the observations at the different epochs will also provide a highly sensitive survey for faint sources including HII regions around relatively low mass stars. The combination of $14 \mathrm{GHz}$ observations at 10 epochs would reach a noise level of $\sim 0.3 \mu \mathrm{Jy}$, sensitive at a 6- $\sigma$ level to the HII regions from stars with masses as low as $6.5 \mathrm{M}_{\odot}$ corresponding to a spectral type of B5 (Diaz-Miller et al. 1998).

\section{Summary}

A SKA time-domain survey of star forming regions will produce well sampled radio lightcurves of hundreds of sources per star forming region. As is the case for infrared light-curves of young sources, these will undoubtably have a range of properties, tracing and constraining a range of phenomena. For example, the identification of rotationally modulated emission will provide the first observational constraints on the rotation rates of embedded protostars. Observations of the 
flickering of HII regions will trace the inflow of material around the highest mass objects while flare activity in these, and other, sources can trace the final infall of material on to the central protostars. These observations can not only constrain the properties of the accretion but also the stochastic heating of the circumstellar regions which can surpress fragmentation (e.g. Krumholz et al. 2007), leading to the formation of more massive stars.

Looking forward to full SKA the factor 10 enhancement in sensitivity will enable studies of smaller (and therefore also shorter time scale) flux variations. Full SKA will also make stellar clusters to beyond the Galactic centre accessible to detailed time-domain studies, probing star formation over a much wider range of environments. The enhanced resolution of SKA will allow studies of the changes in morphology of the sources during their variation in flux, provide stronger constraints on the mechanisms involved and the models for the emission.

Building a comprehensive model for the formation of both low-mass and high-mass stars requires understanding the evolution of gas and dust from molecular clouds down through clumps and cores and eventually on to the forming stars. This is only possible with the combination of ALMA to trace the cool molecular gas and dust and SKA to follow this material into the inner circumstellar regions. The radio light-curves which SKA will produce for hundreds of sources in star forming regions out to $5 \mathrm{kpc}$ will provide the first detailed insight across a wide range of stellar masses of the transient energetic phenomena occurring on the small spatial scales close to the central star. These light curves will for the first time provide a systematic survey which can study the magnetospheric interactions in young binary systems, tracing episodic accretion, the origin of outflows and potentially constrain the rotation rates of deeply embedded sources.

\section{References}

Andre, P., Montmerle, T., Feigelson, E. D., Stine, P. C., \& Klein, K.-L. 1988, ApJ, 335, 940

Anglada, G., Rodriguez, L. F., Canto, J., Estalella, R., \& Lopez, R. 1987, A\&A, 186, 280

Arce, H. G., Mardones, D., Corder, S. A., et al. 2013, ApJ, 774, 39

Balog, Z., Muzerolle, J., Flaherty, K., et al. 2014, ApJ, 789, L38

Bary, J. S., \& Petersen, M. S. 2014, ApJ, 792, 64

Beltrán, M. T., Sánchez-Monge, Á., Cesaroni, R., et al. 2014, A\&A, 571, A52

Bower, G. C., Plambeck, R. L., Bolatto, A., et al. 2003, ApJ, 598, 1140

Brickhouse, N. S., Cranmer, S. R., Dupree, A. K., et al. 2012, ApJ, 760, L21

Brown, A. 1987, ApJ, 322, L31

Casassus, S., van der Plas, G., M, S. P., et al. 2013, Nature, 493, 191

Cesaroni, R., Galli, D., Neri, R., \& Walmsley, C. M. 2014, A\&A, 566, A73

Choi, M., Tatematsu, K., Hamaguchi, K., \& Lee, J.-E. 2009, ApJ, 690, 1901

Cody, A. M., Stauffer, J., Baglin, A., et al. 2014, AJ, 147, 82

De Pree, C. G., Peters, T., Mac Low, M.-M., et al. 2014, ApJ, 781, L36

Diaz-Miller, R. I., Franco, J., \& Shore, S. N. 1998, ApJ, 501, 192

Drake, S. A., Simon, T., \& Linsky, J. L. 1992, ApJS, 82, 311

Dzib, S. A., Rodríguez-Garza, C. B., Rodríguez, L. F., et al. 2013a, ApJ, 772, 151

Dzib, S. A., Loinard, L., Mioduszewski, A. J., et al. 2013b, ApJ, 775, 63

Faesi, C. M., Covey, K. R., Gutermuth, R., et al. 2012, PASP, 124, 1137 
Feigelson, E. D., Carkner, L., \& Wilking, B. A. 1998, ApJ, 494, L215

Feigelson, E. D., \& Montmerle, T. 1999, ARA\&A, 37, 363

Flaherty, K. M., Muzerolle, J., Rieke, G., et al. 2013, AJ, 145, 66

Forbrich, J., Menten, K. M., \& Reid, M. J. 2008, A\&A, 477, 267

Forbrich, J., Preibisch, T., \& Menten, K. M. 2006, A\&A, 446, 155

Forbrich, J., \& Wolk, S. J. 2013, A\&A, 551, A56

Frank, A., Ray, T. P., Cabrit, S., et al. 2014, Protostars and Planets VI, 451

Fuller, G. A., Williams, S. J., \& Sridharan, T. K. 2005, A\&A, 442, 949

Galván-Madrid, R., Peters, T., Keto, E. R., et al. 2011, MNRAS, 416, 1033

Galván-Madrid, R., Rodríguez, L. F., Ho, P. T. P., \& Keto, E. 2008, ApJ, 674, L33

Garay, G., Moran, J. M., \& Reid, M. J. 1987, ApJ, 314, 535

Getman, K. V., Broos, P. S., Salter, D. M., Garmire, G. P., \& Hogerheijde, M. R. 2011, ApJ, 730, 6

Getman, K. V., Feigelson, E. D., Broos, P. S., Micela, G., \& Garmire, G. P. 2008, ApJ, 688, 418

Goedhart, S., Maswanganye, J. P., Gaylard, M. J., \& van der Walt, D. J. 2014, MNRAS, 437, 1808

Gómez, L., Rodríguez, L. F., Loinard, L., et al. 2008, ApJ, 685, 333

Graham, M. F., Meaburn, J., Garrington, S. T., et al. 2002, ApJ, 570, 222

Guedel, M., \& Benz, A. O. 1993, ApJ, 405, L63

Hartmann, L. 2009, Accretion Processes in Star Formation: Second Edition (Cambridge University Press)

Ho, P. T. P., \& Young, L. M. 1996, ApJ, 472, 742

Jørgensen, J. K., Favre, C., Bisschop, S. E., et al. 2012, ApJ, 757, L4

Jørgensen, J. K., Visser, R., Sakai, N., et al. 2013, ApJ, 779, L22

Karim, A., Swinbank, A. M., Hodge, J. A., et al. 2013, MNRAS, 432, 2

Kastner, J. H., Richmond, M., Grosso, N., et al. 2004, Nature, 430, 429

Kirk, H., Myers, P. C., Bourke, T. L., et al. 2013, ApJ, 766, 115

Klassen, M., Peters, T., \& Pudritz, R. E. 2012a, ApJ, 758, 137

Klassen, M., Pudritz, R. E., \& Peters, T. 2012b, MNRAS, 421, 2861

Kounkel, M., Hartmann, L., Loinard, L., et al. 2014, ApJ, 790, 49

Krumholz, M. R., Klein, R. I., \& McKee, C. F. 2007, ApJ, 656, 959

Lee, C. W., Myers, P. C., \& Tafalla, M. 1999, ApJ, 526, 788

Li, Z.-Y., Banerjee, R., Pudritz, R. E., et al. 2014, Protostars and Planets VI, 173

Liu, H. B., Galván-Madrid, R., Forbrich, J., et al. 2014, ApJ, 780, 155

Maercker, M., Mohamed, S., Vlemmings, W. H. T., et al. 2012, Nature, 490, 232

Mann, R. K., Di Francesco, J., Johnstone, D., et al. 2014, ApJ, 784, 82

Massi, M., Forbrich, J., Menten, K. M., et al. 2006, A\&A, 453, 959

Mathews, G. S., Klaassen, P. D., Juhász, A., et al. 2013, A\&A, 557, A132

Meynet, G., Eggenberger, P., \& Maeder, A. 2011, A\&A, 525, L11

Moran, J. M., Garay, G., Reid, M. J., et al. 1983, ApJ, 271, L31

Moscadelli, L., Cesaroni, R., Sánchez-Monge, Á., et al. 2013, A\&A, 558, A145

Muzerolle, J., Furlan, E., Flaherty, K., Balog, Z., \& Gutermuth, R. 2013, Nature, 493, 378

Nazé, Y. 2014, in Putting A Stars into Context: Evolution, Environment, and Related Stars, ed. G. Mathys, E. R. Griffin, O. Kochukhov, R. Monier, \& G. M. Wahlgren, 340-349

Peretto, N., Fuller, G. A., Duarte-Cabral, A., et al. 2013, A\&A, 555, A112 
Pérez, L. M., Isella, A., Carpenter, J. M., \& Chandler, C. J. 2014, ApJ, 783, L13

Pineda, J. E., Maury, A. J., Fuller, G. A., et al. 2012, A\&A, 544, L7

Qi, C., Öberg, K. I., Wilner, D. J., et al. 2013, Science, 341, 630

Rodríguez, L. F., González, R. F., Montes, G., et al. 2012, ApJ, 755, 152

Rodríguez, L. F., Masqué, J. M., Dzib, S. A., Loinard, L., \& Kurtz, S. E. 2014, Rev. Mexicana Astron. Astrofis., 50, 3

Rosen, A. L., Krumholz, M. R., \& Ramirez-Ruiz, E. 2012, ApJ, 748, 97

Salter, D. M., Hogerheijde, M. R., \& Blake, G. A. 2008, A\&A, 492, L21

Stauffer, J., Cody, A. M., Baglin, A., et al. 2014, AJ, 147, 83

Szymczak, M., Wolak, P., \& Bartkiewicz, A. 2014, MNRAS, 439, 407

van der Marel, N., van Dishoeck, E. F., Bruderer, S., \& van Kempen, T. A. 2014, A\&A, 563, A113

van der Marel, N., van Dishoeck, E. F., Bruderer, S., et al. 2013, Science, 340, 1199

Walsh, A. J., Bourke, T. L., \& Myers, P. C. 2006, ApJ, 637, 860

Wolff, S. C., Strom, S. E., Dror, D., Lanz, L., \& Venn, K. 2006, AJ, 132, 749

Zapata, L. A., Loinard, L., Rodríguez, L. F., et al. 2013, ApJ, 764, L14 